\title{
A TEORIA DO FLOW COMO PROMOTORA MOTIVACIONAL PARA ESTUDANTES COM ANSIEDADE MATEMÁTICA
}

\section{THE THEORY OF FLOW AS A MOTIVATIONAL PROMOTER FOR STUDENTS WITH MATHEMATICAL ANXIETY}

\author{
Ana Maria Antunes de Campos ${ }^{1}$
}

\begin{abstract}
RESUMO
A matemática faz parte da vida dos estudantes, desde a infância, ajudando na formação, nas resoluções de problemas e nas atividades cotidianas. No entanto, esse é um tópico que ainda ocasiona estresse e ansiedade. Consequentemente, alguns estudantes apresentam uma resposta negativa aos estímulos numéricos, levando-os a um baixo rendimento escolar específico em matemática. A ansiedade matemática pode afetar negativamente não apenas o comportamento diante da matemática, mas na maneira que os estudantes aprendem a matemática. Uma das reações da ansiedade matemática é a tensão, fuga, tédio, ansiedade, desmotivação, preocupação, sentimento de desamparo e medo frente à matemática. Nesse sentido, este artigo tem como objetivo identificar de que modo os elementos apresentados na Teoria do Flow, podem ajudar no aprendizado da matemática em estudantes que apresentam ansiedade matemática. Foi realizado um estudo bibliográfico que se ocupa em investigar a Teoria do Flow no contexto educacional. Os resultados apontam que os elementos do Flow permitem uma análise diferenciada e individual de como o estudante se apresenta diante as atividades que dependem de suas habilidades, sendo esse um dos elementos mais importante da teoria. A motivação é um processo responsável pela intensidade, direção e persistência de esforços para atingir uma meta, é um fator poderoso que influencia a maneira como os estudantes aprendem e dominam matemática. Isto posto, quando as atividades escolares proporcionam interesse e motivação, os estudantes entram em uma intensa concentração que pode atenuar as reações da ansiedade matemática como desmotivação, desinteresse e tédio.
\end{abstract}

Palavras-chave: Ansiedade Matemática; Flow; Motivação; Dificuldades de Aprendizagem; Educação Básica.

\begin{abstract}
Mathematics has been part of students' lives since childhood, helping with training, problem solving and daily activities. However, this is a topic that still causes stress and anxiety. Consequently, some students show a negative response to numerical stimuli, leading them to a specific low academic performance in mathematics. Mathematical anxiety can negatively affect not only math behavior, but the way students learn math. One of the reactions of mathematical anxiety is tension, flight, boredom, anxiety, demotivation, worry, feeling of helplessness and fear in the face of mathematics. In this sense, this article aims to identify how the elements presented in the Flow Theory can help in the learning of mathematics in students who have mathematical anxiety. A bibliographic study was carried out that investigates the Flow Theory in the educational

${ }^{1}$ Doutoranda em Educação Matemática na Pontifícia Universidade Católica de São Paulo - PUC-SP. São Paulo, SP, Brasil. Endereço para correspondência: Rua Araruna, 75, Apartamento 34N, Bom Clima, Guarulhos, São Paulo, Brasil. CEP: 07196-200. E-mail: camp.ana@hotmail.com.
\end{abstract}

(iD) ORCID iD: https://orcid.org/0000-0003-4276-5776. 
context. The results show that the elements of the Flow allow a differentiated and individual analysis of how the student presents himself in relation to the activities that depend on his abilities, being this one of the most important elements of the theory. Motivation is a process responsible for the intensity, direction and persistence of efforts to reach a goal, it is a powerful factor that influences the way students learn and master mathematics. That said, when school activities provide interest and motivation, students enter an intense concentration that can mitigate the reactions of mathematical anxiety such as demotivation, disinterest and boredom.

Keywords: Mathematical anxiety; Flow; Motivation; Learning difficulties; Basic Education. 


\section{Introdução}

A matemática faz parte da vida dos estudantes, desde a infância, ajudando na formação, nas resoluções de problemas e nas atividades cotidianas. No entanto, esse é um tópico que ainda ocasiona estresse e ansiedade. O mal desempenho em matemática apresentado na sala de aula é um reflexo de uma problemática complexa, que precisa ser investigada para uma ação condizente com a necessidade individual de cada estudante.

Segundo a literatura investigada, a aversão à matemática é conhecida como ansiedade matemática, que é uma resposta negativa perante situações que envolvam a matemática e que modificam o estado cognitivo, fisiológico e comportamental do estudante. (CARMO; SIMIONATO, 2012; MENDES; CARMOS, 2014). Reações como preocupação, ansiedade, desamparo, pânico, esquiva e medo frente à matemática, ocasionam muitas vezes desmotivação, desinteresse, tédio, abandono escolar e fuga de atividades que envolvam a matemática.

Os estudos acerca da ansiedade matemática são em grande parte pesquisas internacionais, nos quais se destacam os autores Dreger e Aiken, (1957); Tobias (1976); Meece, Wigfield e Eccles, (1990); Ashcraft e Kirk (2001). Essas pesquisas são desenvolvidas em áreas científicas distintas, relacionadas à Genética, Psicologia e Neurociência.

Algumas pesquisas (DREGER; AIKEN, 1957; HEMBREE, 1990; CARMO, 2003) apontam que ansiedade matemática se manifesta perante as atividades matemáticas dentre elas: resolução de problemas, avaliações, diante de livros didáticos matemáticos, ao ver uma equação na lousa ou em um papel, ao ouvir o nome do professor de matemática e, ainda, que é dia de aula de matemática. Bem como difere de outras formas de ansiedade, como por exemplo, transtorno de ansiedade, ansiedade geral e a ansiedade social.

Para, Santos et al. (2012), a ansiedade matemática pode levar a erros que interferem na resolução de problemas matemáticos gerando resultados de frustração e aversão, causando um déficit cognitivo que pode ser confundido com a Discalculia. Consequentemente, alguns pesquisadores (DEVINE, 2017; DEVINE et al. 2018) estão estudando essa relação e, até o momento, os resultados sugerem que os distúrbios cognitivos são dissociáveis dos emocionais; a ansiedade pode ser uma reação da discalculia; estudantes com discalculia e ansiedade matemática provavelmente requerem 
diferentes tipos de intervenção. Contudo, segundo os pesquisadores, os estudos não são conclusivos, sendo necessárias futuras investigações.

O constructo da ansiedade matemática sofre impacto da motivação, cognição, emoções e afeto; com o envolvimento dos estudantes na aprendizagem da matemática; papel dos pais e professores. Esses fatores interferem nas decisões dos estudantes de seguirem ou não carreiras que envolvem a matemática.

Baten, Pixner e Desoete (2019) relatam o conhecimento prévio e a inteligência são preditores cognitivos que explicam algumas variações na aprendizagem da matemática. Além disso, afeto e motivação são fatores poderosos que influenciam a maneira como os estudantes aprendem e dominam matemática. A motivação é um processo responsável pela intensidade, direção e persistência de esforços para atingir uma meta, no qual é fundamental distinguir entre a motivação controlada e motivação autônoma.

No qual, a motivação controlada se refere a força que leva o estudante a cumprir uma tarefa, ou seja, uma regulação externa e introjetada, como exemplo: dizer a si mesmo que precisa estudar à tarde, para poder sair com os amigos à noite. A motivação autônoma é encontrar em uma determinada tarefa os aspectos valiosos, ou seja, consiste em regulamentação identificada, regulação integrada, e intrínseca, como exemplo: estudar matemática porque vê a relevância à carreira acadêmica. (BATEN; PIXNER; DESOETE, 2019, p. 461).

O estudo aponta que existe uma relação positiva entre o nível de motivação autônoma e realização em matemática, já a relação entre motivação controlada teve relações negativas significativas com o desempenho acadêmico. A ansiedade matemática influencia na motivação dos estudantes e nas competências matemática a longo prazo.

Nesse sentido, essa pesquisa tem como objetivo identificar de que modo os elementos apresentados na Teoria do Flow, elaborado por Mihaly Csikszentmihalyi, podem ajudar no aprendizado da matemática em estudantes que apresentam ansiedade matemática. Foi realizada uma pesquisa bibliográfica que se ocupa em investigar a Teoria do Flow no contexto educacional. 


\section{Teoria do Flow}

A Teoria do Flow foi desenvolvida pelo psicólogo húngaro Mihaly Csikszentmihalyi na década de 1960. Seus estudos procuravam entender o tipo de atividade que permitia a diversão e prazer, envolvendo profundamente as pessoas, levando-as a perda da noção do tempo, desprezando desconfortos como a fome e a fadiga e tornando-se uma atividade motivadora por si só, sem recompensa extrínseca.

Segundo Csikszentmihalyi (2014) a experiência de fluxo é de profunda concentração em um conjunto limitado de estímulos que são aceitos pela pessoa como relevantes, ou seja, o instante em que uma pessoa está totalmente concentrada e absorta em uma atividade. Nessa circunstância, há equilíbrio entre a capacidade e o desafio, a noção de tempo é alterada, a sensação de controle é modificada, deixando de ser controlado para passar a controlar suas ações e ambiente. Para ele, as atividades como artes, esportes, jogos e outras atividades de hobbies, fornecem um ambiente onde a curiosidade e o desafio servem de motivação e facilitam a concentração e o envolvimento com a atividade.

Csikszentmihalyi (1990) define oito elementos que estão presentes no flow, a saber:

1. Metas e submetas são definidas - durante a imersão ao Flow, a pessoa sabe o que deve ser feito para que os objetivos sejam alcançados. As metas e submetas são claras e bem definidas.

2. Feedback é imediato - a pessoa sabe que, independentemente das situações externas, se está apresentando um bom desempenho para que os objetivos sejam atingidos, ou seja, se está sendo autossuficiente para desenvolver a atividade que se propôs.

3. O presente é primordial - no Flow, a pessoa se concentra naquilo que está fazendo, não permitindo que as memórias do passado e as inquietações do futuro interfiram na atividade que está desempenhando.

4. A noção de tempo - o estado de fluxo interrompe nossa percepção de tempo, fazendo com que períodos longos se pareçam com instantes. Quando estamos imersos num processo criativo, por exemplo, podemos nos surpreender ao olharmos um relógio e descobrirmos as horas já avançaram.

5. Concentração profunda - imersão total na atividade, a discriminação entre pensamento e ação não estão presentes no processo. 
6. Controle - a pessoa tem perceptibilidade dos objetivos a serem alcançados e possuem controle sobre a situação em que se encontram e onde ambicionam chegar.

7. Equilíbrio entre capacidade e oportunidade - o sentimento de confiança em si mesmo se expressa na percepção das oportunidades nos preparando à ação.

8. Exclusão do ego - o envolvimento com a atividade é maior do que o ego, ou seja, o que importa é atingir o objetivo e não o reconhecimento por desenvolver a tarefa.

Csikszentmihalyi (2014) expõe que os estudantes aprensentam dificuldades de aprendizagem não por problemas cognitivos, mas por probelmas afetivos, emocionais e motivacionais. O Flow ajuda no processo de aprendizagem, uma vez que permite uma análise diferenciada e individual de como o estudante se apresenta diante as atividades que dependem de suas habilidades. Sendo esse um dos elementos mais importante da Teoria.

Segundo Csikszentmihalyi (1990) existe uma linha tênue entre a ansiedade, tedio e Flow, que pode ser observada na figura 1.

Figura 1 - Esquema do Flow

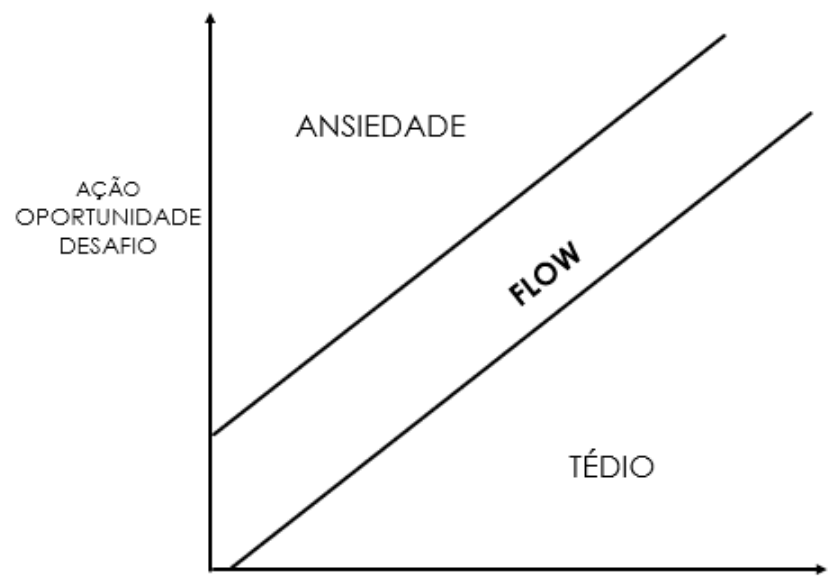

Fonte: (CSIKSZENTMIHA LYI, 1990, p. 74)

Quando o desafio é muito maior do que as habilidades ou as oportunidades são muito maiores do que a capacidade da pessoa, é possível esperar como resultado a ansiedade; se as habilidades superam desafios e o autocontrole, aparece o tédio; quando as habilidades e os desafios se equilibram, a situação geralmente permite a vivência do estado de Flow. (CSIKSZENTMIHALYI, 2014). 
Para o autor, o desafio dá ao estudante, visão e direção, foco e perseverança; o objetivo está contido no processo de aprendizado e esse aprendizado intrinsecamente motivado pode ser autotélico, ou seja, é buscado por si só.

Ao atingir o estado de Flow a pessoa está em total concentração, interesse, motivação e criatividade." Essa intensa concentração é sustentada em parte pelo fato de a atividade ter objetivos claros e fornecer feedback claro às ações da pessoa" CSIKSZENTMIHALYI, 2014, p. 6).

$\mathrm{Na}$ escola, existem várias distratores e elementos que tiram o foco e atenção dos estudantes. Do mesmo modo, existem disciplinas que podem gerar certo medo, receio e ansiedade, dentre elas à matemática. Nesse sentido, observa-se que a Teoria do Flow pode ser utilizada em sala de aula, em especial na educação matemática.

Csikszentmihalyi (2014) constatou que a atenção dos estudantes pode estar relacionada ao estado de Flow do professor em quase todas as disciplinas, contudo em matemática essa relação deixa de ocorrer depois da quarta série, em que a maioria dos estudantes estão tão distraídos e não prestam atenção, mesmo que o professor esteja no estado do Flow, em decorrência esses estudantes deixam de ser bons em matemática.

Os estudantes encontram recompensas intrínsecas muito mais facilmente na arte e na música do que na matemática. $\mathrm{O}$ fato de o estudante não apreciar a disciplina dificulta o desenvolvimento de altos níveis de proficiência. Assim como a falta de prazer no ensino, que pode privar a atividade de seu principal valor, a importância da motivação intrínseca. (CSIKSZENTMIHALYI, 2014),

De acordo com os estudos de Csikszentmihalyi (2014, p. 438) é fundamental considerar dois tipos de motivações no âmbito escolar:

[...] baseado na expectativa de recompensas de longo prazo (por exemplo, cumprimento da carreira) expectativas ou necessidades psicológicas e uma baseada nas recompensas de experiência (por exemplo, desfrutando da própria atividade). O primeiro tipo de motivação pode ser extrínseco ou intrínseco e o segundo tipo de motivação, baseado na experiência imediata, sempre tende a ser intrínseca, na medida em que as recompensas são inerentes à própria atividade.

Para Csikszentmihalyi (2014) a motivação intrínseca desempenha um importante papel na decisão de um estudante fazer ou não cursos mais avançados de matemática. Segundo o autor, os estudantes apontam que as aulas de matemática e as ciências da 
computação são os assuntos mais intensos, desafiadores e relevantes, contudo não são necessariamente os mais motivadores.

\section{Principais evidências}

A ansiedade matemática pode estar associada a fatores emocionais, afetivos e motivacionais, no qual alguns estudantes desenvolvem atitudes negativas quanto a matemática (GEIST, 2010).

Na Teoria do Flow (Csikszentmihalyi, 1999), as pessoas são afetadas por condições externas, como por exemplo por meio da interação com outras pessoas e em locais que frequentam, contudo o que importa para atingir o estado de Flow é o que fazemos e como reagimos a essas experiências. Nesses experimentos é que vivenciamos desafios que "exigem habilidades, concentração, criatividade e satisfação." (CSIKSZENTMIHALYI, 1999, p. 62). Esses fatores modificam o comportamento dos estudantes permitindo a auto realização e auxilia na compreensão do processo de aprendizagem.

Csikszentmihalyi (1999) divulga que quando as pessoas percebem que os desafios vão além de suas capacidades, elas procuram evitar essas situações, logo para apreciar atividades complexas é necessário um investimento inicial de atenção, tornando-a agradável, só após esse processo, é que será possível envolver-se com à atividade

Em sala de aula, as atividades devem ser elaboras a partir dos elementos do Flow, com vista a permitir: disciplina, situações de alto desafio, concentração, criatividade, satisfação, motivação, meta claras, regras de desempenho, feedback, que permitam uma quantidade variável de controle (CSIKSZENTMIHALYI, 1999).

Os elementos da teoria do Flow estão relacionados na discussão acerca da ansiedade matemática, como: a noção de estar no controle; a perda da consciência contrastada com a falta de autoconsciência; erros do passado que afetam os objetivos, prejudicando a atenção e interferindo na concentração profunda; feedback imediato, ou seja, receber um retorno positivo, bem como compreender como melhorar sua experiência de domínio; metas claras, fornece instruções que envolvam os estudantes em um desafio que pode ser alcançado de acordo com as suas habilidades. 


\section{Tecendo algumas considerações}

O aprendizado da matemática acontece como uma escada ou corrente, ou seja, é necessário conhecimentos anteriores para prosseguir em seus estudos. Os estudantes devem estar totalmente concentrados para compreenderem as questões matemáticas, no entanto, alguns iniciam os estudos ansiosos, não conseguindo sair desse estado.

A Teoria do Flow possibilita que os estudantes vivenciem situações prazerosas, no qual desenvolvam a concentração, o interesse, a motivação, a criatividade e, consequentemente, o aprendizado.

As experiências do Flow permitem o desenvolvimento de habilidades necessárias para o aprendizado da matemática, amparando os estudantes com ansiedade matemática na reflexão sobre suas habilidades e os desafios proposto pela matemática.

A ansiedade matemática pode estar associada aos conceitos, operações e na resolução de problemas que, algumas vezes não são interpretados e relacionados com a vida cotidiana. A Teoria do Flow foi desenvolvida com vistas a compreender em que áreas e situações as pessoas se concentram totalmente, esquecendo de fatores externos.

Nesse sentido, se conjectura que ela pode ser atenuada a partir dos elementos do Flow, visto que estão associados com a ação, desafio, regras, metas claras, feedback, organização, imaginação, criatividade, motivação e ao desenvolvimento do raciocínio.

As atividades em sala de aula devem ser elaboradas como um instrumento para aprimorar as habilidades necessárias para a construção do saber matemático, possibilitando o trabalho em grupo, o senso crítico, a argumentação, o espírito investigador e a partir dessa premissa, ajudar na compreensão de conceitos relacionados ao ensino da matemática.

Isto posto, se conjectura que os efeitos negativos da ansiedade matemática podem ser atenuados por atividades que contemplem os elementos da Teoria do Flow, possibilitando a concentração total, motivação e criatividade, um dos caminhos para o aprendizado da matemática.

Observa-se como uma limitação desse artigo o corpus de investigação quanto as pesquisas acerca da Teoria do Flow e a ansiedade matemática. As reflexões sobre ansiedade matemática, motivação e Flow possibilitam futuras pesquisas no âmbito do processo de ensino e aprendizagem da matemática, voltando o olhar para práticas pedagógicas que impulsionam o diálogo na educação matemática. 


\section{Agradecimentos}

Agradecemos à Coordenação de Aperfeiçoamento de Pessoal de Nível Superior (CAPES) e à Pontifícia Universidade Católica de São Paulo - PUC-SP pelo apoio oferecido ao desenvolvimento da pesquisa aqui apresentada.

\section{Referências}

ASHCRAFT, M. H.; KIRK, E. P. The relationships among working memory, math anxiety, and performance. Journal of Experimental Psychology: General, v. 130, N. 2, p.224-237, 2001.

BATEN, E.; PIXNER, S.; DESOETE, A. Motivational and Math Anxiety Perspective for Mathematical Learning and Learning Difficulties. In: FRITZ, A.; HAASE, V. G.; RÄSÄNEN, P. (Editors). International Handbook of Mathematical Learning Difficulties: From the Laboratory to the Classroom, Springer International Publishing AG, p. 557-568, 2019.

CARMO, J. S; SIMINOATO, A. M. Reversão de ansiedade à matemática: alguns dados da literatura. Psicologia da Educação, v. 17, n. 2, p. 317-327, jun., 2012.

CARMO, J. S. Ansiedade matemática: conceituação e estratégia de intervenção. In: BRANDÃO, M. Z. da S., CONTE, F. C. de S., BRANDÃO, F. S., INGBERMAN, Y. K., MOURA, C. B. de, SILVA, V. M.; OLIANE, S. M. (Orgs.). Sobre comportamento e cognição: A história e o avanços, a seleção por consequências em ação. Santo André: Esetec, v. 11, p. 433-442, 2003.

CSIKSZENTMIHALYI, M. Flow: the psychology of optimal exeperience. 1st ed. Harper Perennial Modern Classiscs, 1990.

\section{A descoberta do fluxo: a psicologia do envolvimento com a vida} cotidiana. Rio de Janeiro. Rocco, 1999.

Applications of Flow in Human Development and Education. The Collected Works of Mihaly Csikszentmihalyi. Springer International Publishing, 2014.

DEVINE, A.; HILL, F.; CAREY, E.; SZÜCS, D. Cognitive and emotional math problems largely dissociate: Prevalence of developmental dyscalculia and mathematics anxiety. Journal of Educational Psychology, 110(3), p.431-444, 2018.

DEVINE, A. Cognitive and emotional mathematics learning problems in primary and secondary school students. 248f. Dissertation for the degree of Doctor of Philosophy, University of Cambridge, 2017. 
DREGER, R. M.; AIKEN, L. R. The identification of number anxiety in a college population. Journal of Educational Psychology, v. 48, p. 344-351, 1957.

GEIST, Eungene. The Anti-Anxiety Curriculum: Combating Math Anxiety in the Classroom. Journal of Instructional Psychology, v. 37, n.1, p. 24-31, mar., 2010.

HEMBREE, R. The nature, effect, and relief of mathematics anxiety. Journal for Research in Mathematics Education, v. 21, p. 33-46, 1990.

MEECE, J.L.; WIGFIELD, A.; ECCLES, J. S. Predictors of math anxiety and its influence on young adolescents' course enrollment intentions and performance in mathematics. Journal of Educational Psychology, v. 82 (1), p. 60-70, feb., 1990.

MENDES, A. C; CARMO, J. S. Atribuições dadas à matemática e ansiedade ante a matemática: o relato de alguns estudantes do ensino fundamental. Bolema, v. 28, p. 368, dez. 2014.

SANTOS, F. H.; SILVA, P. A.; RIBEIRO, F. S.; DIAS, A. L. R. P.; FRIGÉRIO, M. C.; DELLATOLAS, G.; ASTER, M. V.Number processing and calculation in Brazilian Children Aged 7-12 years. The Spanish Journal of Psychology, v. 15, n. 2, 2012.

TOBIAS; S. Succeed with Math: Every Student's Guide to Conquering Math Anxiety. College Entrance Examination Board, 1987.

Recebido em: 05 /02 / 2021 Aprovado em: 18 / 04 / 2021 